\title{
Synthesis of Strong Sweetener Sucralose
}

\author{
Ye Luo, Lei Xu \& Xiaofei Sun \\ College of Pharmacy, Soochow University \\ Suzhou 215123, China \\ Tel: 86-512-6588-0413Ｅ-mail: sunxiaofei@suda.edu.cn
}

\begin{abstract}
Synthesize sucralose from sucrose as a starting material by following steps: first synthesis sucrose-6-acetate, and then by chlorination, at last deacetylation. The Synthetic Approach is simple, with high yield and under mild reaction conditions. the ultimate product sucralose was purified and bleaching, and its structure was characterized by the IR, MS, etc.
\end{abstract}

Keywords: Sucrose-6-acetate, Sucralose, Synthesis

The sucralose is TGS for short, its chemical name is $1^{\prime}, 6^{\prime}$ - dichloro - $1^{\prime}, 6^{\prime}$-didesoxy - $\beta$-D - fructofuranose - 4chloro - 4- desoxy - $\alpha$-D - fructofuranose, is one derivative of the halogeno sucrose. It's mild and mellow, with aromatic flavor and stable property, it's low in calories and good for consumers' health; it can be used together with the traditional sweetener, and used independently. it's good in sweet taste and mouth feel (Kang, 2001, pp. 12). However, the sucralose can't rot teeth away, and not cause many diseases as the fructose and maltose, besides its low sugar price is not so high as other nutritional sweeteners(Huang, 2000, pp. 22),moreover, the toxicology and the safety test indicated: sucralose does not have teratogenicity, discontinuity, reproduction toxicity, cancerous as well as nerve toxicity, also does not have any effects on animal's blood sugar level and the insulin secretion (Gao, 2004, pp. 5).Therefore, it could be edible for obese patients, patients with cardiovascular disease and diabetes. It is a strong sweetener prepared by British Professor Hough L in the late 1970s (Yan, 2004, pp 165), it's also artificially developed, and the most competitive, non-nutrition, perfect high-intensity sweetener until now (Tian, 2006, pp. 3).

The demand for sucralose in China is about 50 tons annually, the majority depends on imports, and is only used in some relatively high-grade food at present, it hasn't been widely used in China yet(Shen, 2007,pp. 137).Therefore, if the basic research on the sucralose production technology can be strengthened, that will further improve the technique of production, reduce the cost of product, and extend its application range greatly, and thus give an impetus to the development of food additive industry in China. Nowadays, people have explored many methods of the development about sucralose, such as the tritylation method, the monoesterification, biosynthesis, the hydrolyzing raffinose and the chlorination of sucrose derivative .etc (Chen, 2007, pp. 90). The chemical synthesis method above always take too many steps and the technical process is complex. The fermentation of chemically enzymatic synthesis is complex, and it's difficult to purify the intermediary product, with high cost. The monoesterification only needs three steps in the reaction of synthesis, with high yield and low cost, the intermediary product is easy to separate, so it's an ideal technical process for the synthesis of sucralose (Kang, 2006, pp. 59).

We synthesize sucralose from sucrose as a starting material by following steps: first synthesis sucrose-6-acetate, and then by chlorination, at last deacetylation (just as the following figure shows), and make research on technological process, reaction conditions, etc.

\section{Experimental material and equipments}

\subsection{Chemical materials and reagents}

Sucrose, DMF, trimethyl orthoacetate, tert-Butylamine, p-TsOH,1, 1, 2 trichlorethane, thionyl chloride and etc are analytical pure or chemical pure.

\subsection{Equipments}

RE-2000 Rotary Evaporator (made in Shanghai Yarong Biochemistry Instrument Factory); 85-2A Magnetic Stirrer (made in Jintan Fuhua Instrument Co., Ltd); SHZ-DA Vacuum Pump of Circulating Water(made in Gongyi Yingyu Yuhua Instrument Factory); DZF-6050 Vacuum Drying Oven(made in Shanghai Jinghong Laboratory Instrument Co., Ltd); XT4A Microscopic Melting-point Detector(made in Beijing Keyi Electron Optical Instrument Factory); ProStar LC240 Infrared Spectrophotometer and Saturan2200 mass spectrometer(both made in the US Varian,Inc). 


\section{Experimental methods and results}

\subsection{Synthesis of sucrose-6-acetate}

Take sucrose $(25.0 \mathrm{~g})$ to anhydrous DMF $(100 \mathrm{ml})$, the reaction mixture is stirred under the room temperature, and trimethyl orthoacetate $(10.5 \mathrm{ml})$ is added dropwise slowly, and $\mathrm{p}-\mathrm{TsOH}(0.15 \mathrm{~g})$ is added, it's stirred for 2.5 hours, then add distilled water $(10 \mathrm{ml})$, keep reaction for 40 minutes again, after that, tert-Butylamine is added, the reaction mixture continues to be stirred for 1.5 hours, when it's finished, concentrate the reactant, add ethyl acetate and methanol, stir the mixture under the heating condition, cool down, at last white powdery crystal $(12.8 \mathrm{~g}, 51 \%$.) is separated out. mp 93 95 ${ }^{\circ} \mathrm{C}$, IR $\left(\mathrm{KBr}, \mathrm{cm}^{-1}\right): 3416(-\mathrm{OH}), 1728(\mathrm{C}=\mathrm{O})$.

\subsection{The chlorination of sucrose - 6- acetate}

Take sucrose - 6- acetate $(10 \mathrm{~g})$ to dissolve in anhydrous DMF $(100 \mathrm{ml})$, under the ice-bath condition, add dropwize the mixed solvent of thionyl chloride $(23 \mathrm{ml})$ and 1,1,2- trichlorethane $(43 \mathrm{ml})$ slowly, when it's finished, maintain the low temperature in 30 minutes; heat up to $115^{\circ} \mathrm{C}$ slowly within 2 hours, reflux in 1.5 hours; and then cool off the reaction liquid until the temperature is below $15^{\circ} \mathrm{C}$, add weak aqua ammonia $(6 \mathrm{~mol} / \mathrm{L}, 20 \mathrm{ml})$ dropwise slowly, and keep temperature below $30^{\circ} \mathrm{C}$, it is neutralized with the alkaline solution, The organic phase is separated from the aqueous phase, extract the aqueous phase with $1,1,2$ trichlorethane $(20 \mathrm{ml} \times 3)$, the organic phase is combined, and the solvent was evaporated under the diminished pressure, thick product $(5.9 \mathrm{~g}, 59 \%$.) is obtained.

\subsection{The preparation of sucralose}

Sucrose-6-acetate $(5 \mathrm{~g})$ dissolves in methanol $(28.1 \mathrm{ml})$ which contains sodium methoxide $(0.11 \mathrm{~g})$, under room temperature, diminished pressure condition, the reaction mixture is stirred for 2 hours, the dark-brown crude product is obtained by suction filtration, and then recrystallized with methanol, the product is decolored by active carbon, sucralose $(3.6 \mathrm{~g}, 70 \%)$ is obtained after vacuum drying. mp83 85 ${ }^{\circ} \mathrm{C}, \mathrm{IR}\left(\mathrm{KBr}, \mathrm{cm}^{-1}\right): 3452(-\mathrm{OH}), 1086(\mathrm{C}-\mathrm{O}), 732(\mathrm{C}-\mathrm{Cl}) \cdot \mathrm{MS}(\mathrm{m} / \mathrm{z}): 397\left(\mathrm{MH}^{+}\right), 197\left(\mathrm{MH}^{+}-\mathrm{C}_{6} \mathrm{H}_{9} \mathrm{Cl}_{2} \mathrm{O}_{3}\right)$.

\section{Discussion}

When it comes to the catalytic reaction with $\mathrm{p}-\mathrm{TsOH}$, if the quantity of the acid is insufficient, the solution $\mathrm{PH}$ value would be high, and the reaction won't be complete, plenty of sucrose have not participated in the reaction, it would affect the yield of sucrose-6-acetate directly. If $\mathrm{p}$ - $\mathrm{TsOH}$ is excessive, it would make the product break down. if the quantity of tert-Butylamine is insufficient, the $\mathrm{PH}$ value of solution is low, the reaction would not be complete.

Since DMF is also used for solvent during the chlorination of sucrose - 6- acetate, after obtaining the sucrose - 6acetate in the reaction, the solid intermediate may not be obtained directly, it only need provide sucrose - 6- acetate in DMF solution for the chlorination In the process of sucralose's preparation, the chloride reaction is the key to the entire synthesis process. when thionyl chloride is added, the reaction temperature needs to be controlled, because a quantity of heat will be emitted at this time, the product is prone to charring. In order to control the temperature, this ice-bath was used, it could keep the reaction temperature low, be beneficial for the reaction to carry on smoothfully. The mixture of thionyl chloride and 1,1,2- trichloroethane is added dropwise with the dropping funnel, otherwise heat would be emitted too quickly in reaction mixture, the temperature can't be diminished in time, it would have negative effect on the experiment.

We used the method of group protected in our experiment, carried on improvement to the existing preparation method, the product is obtained only through three-step reaction, each step is easy to operate, the raw material is easily obtained, increasing the yield of the product, reducing the production costs greatly and being more helpful for the industrial production.

\section{Acknowledgement}

The authors would like to acknowledge College of Pharmacy and The Center of Analysis and Measurement (CAM) at Soochow University for their sponsorship of this work.

\section{References}

Kang, W. M. (2001). The Characters and Applications of The Sucralose. Beverage \& Fast Frozen Food Industry, 1(4), pp. 12

Huang, S. M., Zhang, H.G., \& Li, S.F. (2000). Progress in Research and Application of High-intensity Sweetener Sucralose. Chemical Industry and engineering Progress, 19(4), pp. 22

Gao, J. Y., Chen, H. B (2004). Application of Sucralose in Sugar Free Chocolate and Chewing Gums. The food industry, (6), pp. 5

Yan, R.A., L,Y. (2004). Study on the Synthesis of Sucralose. Chemical World, 45 (3), pp. 165

Tian, T. N. , Tian, Y. Z. ( 2006). The Advance of Synthetic Technology Study on the Sweetener Sucralose. Hebei 
Chemical, 29 (5), pp. 3

Shen, Y. F. . Ma, Z. Z. , \& Li, H. (2007). The Characteristic of Sucralose and Its Application in Food. China Food Additives, 29 (4), pp. 137

Chen, J. E. (2007). Development of Super Edulcorant -- Trichlorosaccharose. Liquor-Making Science \& Technology, (2), pp. 90

Kang, W. T. , Yang, J. M. , \& Chen, Q. etc. (2006). The Study of New Kind of Sweetener Sucralose, China Condiment, (1), pp. 59

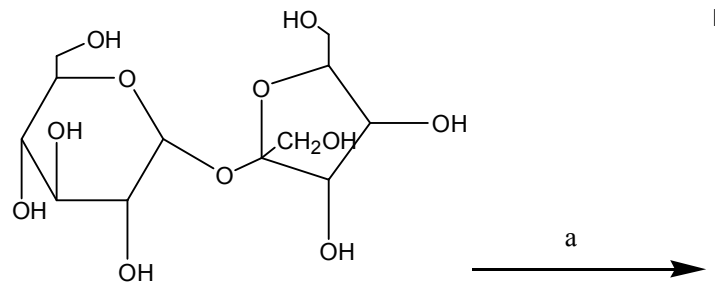

sucrose

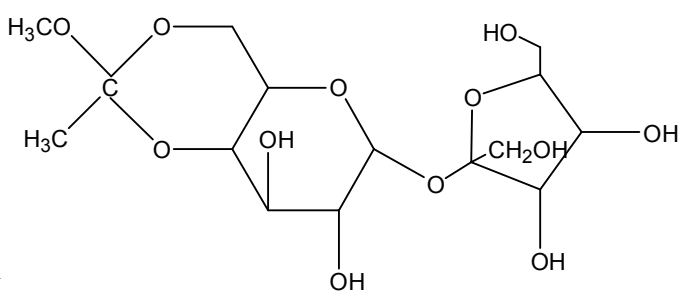

intermediate

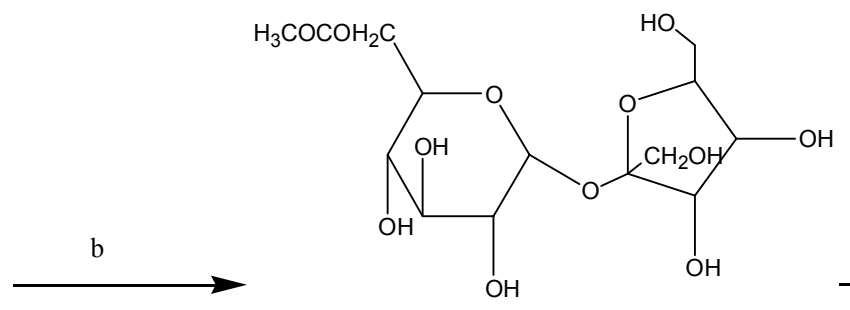

sucrose-6-acetate

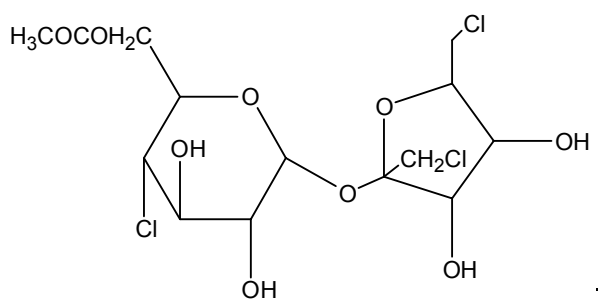

$4,1^{\prime}, 6^{\prime}$-trichlorosucrose-6-acetate

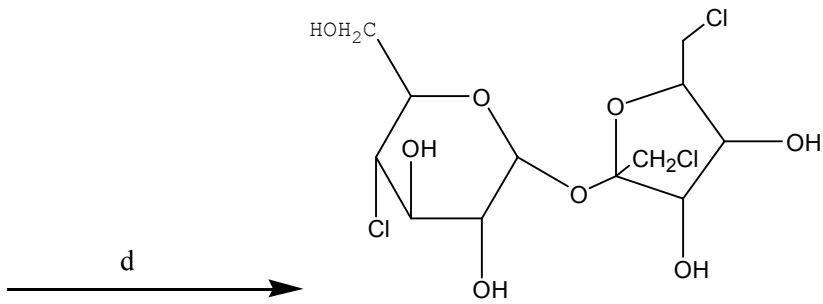

sucralose

Figure 1. Technical process for synthesizing sucralose: (a)p-TsOH, trimethyl orthoacetate, rt, 2.5h; (b) water, tert-Butylamine, rt 1.5h; (c)1,1,2- trichlorethane, $\mathrm{SOCl}_{2} 0^{\circ} \mathrm{C}, 30 \mathrm{~min}$; (d) $\mathrm{CH}_{3} \mathrm{OH}, \mathrm{CH}_{3} \mathrm{ONa}$, rt, $2 \mathrm{~h}$ 